\title{
Monosodium lodoacetate Induced Subchondral Bone Microstructure and Inflammatory changes in an Animal Model of Osteoarthritis
}

\section{Zheming Bao}

960th Hospital of PLA

Mengli Chen ( $\square$ hellolily301@163.com )

960th Hospital of PLA https://orcid.org/0000-0002-6035-0905

Chen Li

Chinese PLA General Hospital

Qing Shan

Chinese PLA General Hospital

\section{Yichen Wang}

Chinese PLA General Hospital

\section{Wenshan Yang}

Chinese PLA General Hospital

Research article

Keywords: Animal model, Osteoarthritis, Bone microarchitecture

Posted Date: June 7th, 2021

DOI: https://doi.org/10.21203/rs.3.rs-516360/v1

License: (1) This work is licensed under a Creative Commons Attribution 4.0 International License.

Read Full License 


\section{Abstract}

Objective: To verify the presence of histopathological injury and cartilage degeneration in MIA induced joints. To investigate the correlation between inflammatory reaction and subchondral bone remodeling in a rodent osteoarthritic model.

Method: Intra-articular injection of MIA was performed in Wistar rats to induce OA. After 4 weeks intervention, the animals were sacrificed, changes in intrinsic structural properties of the subchondral bones were measured using micro-CT scanning. Moreover, the histological evaluation and biochemical analysis was conducted using histochemical staining, immunofluorescence, real-time polymerase chain reaction, and western blot analysis.

Results: This study demonstrated that intra-articular injection of MIA increased chondrocyte apoptosis and promoted cartilage matrix degradation, such as cartilage surface defects and shallow or even disappeared staining. MIA also induced the inflammatory process, improved the expression of IL-1 $\beta$, TNFa, matrix metalloproteinase, and decreased the expression of cartilage specific proteins with the extension of modeling time. Meanwhile, the MIA also significantly accelerated the subchondral bone remodeling at both week two and week four, as shown by the decreased subchondral bone density, thinning of trabeculae, disordered cartilage structure and morphology.

Conclusion: Intra-articular injection of MIA can lead to articular cartilage degeneration and subchondral bone remodeling in the rat model of OA. MIA induced rodent osteoarthritic model would cause decreased subchondral bone density, sparse trabecular bone, and other manifestations of osteoporosis accompanied by an inflammatory response, which would worsen with the progression of modelling time.

\section{Introduction}

Osteoarthritis $(\mathrm{OA})$, a degenerative joint condition, is characterized by progressive degeneration of articular cartilage, subchondral bone remodeling, inflammation and pain ${ }^{[1]}$. OA is especially initiated by excessive mechanical loading on the weight-bearing joints, leading to functional disability, breakdown of the articular cartilage, and economic stress for both the individual and government ${ }^{[2]}$.

Proinflammatory cytokines and inflammatory mediators play a vital role in the pathogenesis of OA. Multiple studies have shown that OA patients contained elevated levels of inflammatory mediators ${ }^{[3]}$. Interleukin-1 beta(IL-1 $\beta$ ) and tumor necrosis factor alpha (TNF- $\alpha$ ), as the principal proinflammatory mediators, induce the production of matrix metalloproteinases (MMPs), potentially resulting in downregulation of collagen-II and aggrecan in articular cartilage ${ }^{[4,5]}$. When the self-perpetuating inflammation and cartilage wearing process overwhelms the reparative capacity of the chondrocytes, cartilage thinning and $\mathrm{OA}$ tend to occur ${ }^{[5,6]}$.

Another aspect of OA often overlooked is the alternation in the subchondral bone compartment. Articular cartilage has always been the main target of basic research on $\mathrm{OA}$, but there are still abundant data 
which indicate an important role of the subchondral bone in the pathogenesis of $O A^{[7,8]}$. Increased osteoclastic activity and bone resorption are often observed in the subchondral bone during the early stages of OA. Furthermore, the anabolic and catabolic processes of bone tissue were manifested unbalanced in osteoarthritic subchondral bon ${ }^{[9]}$, while producing various cytokines which seep through the bone-cartilage interface, initiate chondrocyte hypertrophy and promote cartilage degradation ${ }^{[10]}$. All in all, osteoarthritic subchondral bone would influence the integrity of the overlying cartilage biologically and mechanically ${ }^{[11]}$.

In-vivo osteoarthritic rat model is commonly induced by monosodium iodoacetate (MIA), a glyceraldehyde-3-phosphate dehydrogenase(GAPDH) inhibitor that simulates the phenomena observed in human $\mathrm{OA}$ such as inflammatory response ${ }^{[3,12]}$, progressive loss of chondrocytes and lesions in the cartilage $^{[1,2,13,14]}$. Although numerous studies have used MIA as an inducer in animal models of knee $\mathrm{OA}^{[15]}$, the relationship between MIA induced articular cartilage degeneration and changes in the expression of inflammatory factors after joint damage remains ambiguous.

As local inflammation plays an integral part in the pathogenesis of $O A$, we hypothesized that different phases of MIA induced OA may be associated with the changes of subchondral bone microstructure and the progression of local inflammation. Therefore, the present study aimed to investigate the expression of inflammation-associated cytokines and characteristics of articular cartilage in the joints of the osteoarthritic rat model induced by MIA. In the meantime, bone histomorphological remodeling in the subchondral bone microstructure during the progression of OA was observed in in vivo settings.

\section{Materials And Methods}

\section{Animals}

For this experiment, we used 36 specific pathogen-free male Wistar rats, weighing 240-260 g, obtained from SPF (Beijing) Biotechnology Co.,Ltd. (Beijing, China). The rats were housed in plastic cages at room temperature $\left(21-24^{\circ} \mathrm{C}\right)$ in a $12 \mathrm{~h}$ light and dark cycle with free access to food and water. All experimental animals were fed in the laboratory animal center of Chinese PLA General Hospital. The experiments were performed in line with the recommendations of the Ethics Committee of Chinese PLA General Hospital, which approved the experimental protocol (Approval numberSQ2020127).

The rats were randomly and equally divided into three groups: (a) sham group ( $n=12)$ where the animals received sterile saline $0.9 \%$ for four weeks; (b) MIA two weeks group $(n=12)$ where the animals received MIA for two weeks; (c) MIA four weeks group $(n=12)$ where the animals received MIA for four weeks.

\section{Induction of knee OA}

The MIA-induced OA rat model was used to investigate the correlation between joint cartilage degeneration and inflammatory expression in $O A$. To induce $O A$, Wister rats were anesthetized by intraperitoneal injection of $3 \%$ pentobarbital sodium $(45 \mathrm{mg} / \mathrm{kg})$ and received a single intra-articular 
injection of $50 \mu$ l solution into the right knee joint cavity using a microsyringe (26G). Rats in the MIA group was intra-articular injected with $50 \mu \mathrm{L}$ of $3 \mathrm{mg} \mathrm{MIA}$, which were diluted in sterile saline $0.9 \%$. On the day of injection, the solution of MIA, which was purchased from Aladdin (Shanghai, China), was freshly prepared in sterile saline $0.9 \%$ at $60 \mathrm{~g} / \mathrm{L}$ concentration. The animals of the sham group received an intra-articular injection of an equivalent volume of saline.

Rats were placed in the prone position. The limbs of the rat were gently strapped with hemp ropes on an operating table. The leg was flexed keeping the knee at a $90^{\circ}$ angle and the needle was positioned through the infrapatellar ligament. In the sham group, the same surgical procedure was performed.

After one-week adaptive breeding, the sham and MIA four weeks group was injected in the second week for four weeks. The MIA two weeks group adapted the treatment in the fourth week for two weeks.

Rats were sacrificed by pentobarbital overdose after four weeks following adaptive breeding. The knee joint specimens of the distal femur were isolated and stored in liquid nitrogen for further experiments, while proximal tibia samples were harvested for histological assessment.

\section{Histological analysis}

Histological changes were analyzed to assess the effect on cartilage degeneration in the knee joint of MIA-induced OA rats. The right knees of the rats were dissected and soft tissues were removed from the tibial-femoral joints. The proximal tibia was fixed in $4 \%$ paraformaldehyde for 48 hours and then transferred to $10 \%$ ethylenediaminetetraacetic acid for decalcification, with the solution being replaced every 5 days for 4 weeks ${ }^{[16]}$. Afterwards, the joint tissues were processed into paraffin blocks and sectioned on a coronal plane at the thickness of $5 \mu \mathrm{m}$ using a microtome (Leica RM2016, Shanghai, China). These samples were subsequently processed for histopathology and stained with hematoxylin and eosin (H\&E), safranin fast green and toluidine blue. Tibial cartilage and subchondral bone below the articular cartilage was evaluated using an OOCHAS (Osteoarthritis Cartilage Histopathology Assessment System) score method ${ }^{[17]}$. All of these histological evaluation procedures were performed by a blinded pathologist. The aspects of the joint evaluated were OA depth progression into cartilage, extent of joint involvement. Semi-quantitative method was used to produce an OA score, an index of combined grade and stage with a range of $0-24^{[17]}$.

All slides were observed and analyzed using a light microscope (Nikon Eclipse Ti2, Tokyo, Japan) equipped with an imaging software (NIS elements, Tokyo, Japan). At least three slides were evaluated for each tissue sample.

\section{Micro computed tomography (micro-CT) scanning}

The microarchitectural deterioration in MIA induced OA models was conducted by micro-CT (Quantum GX, PerkinElmer, Kansas, USA). The formalin-fixed tibial bones were placed vertically and flat in the sample tank so that the longitudinal axis of the specimen was perpendicular to the ray. The scanning voltage was set at $70 \mathrm{kV}$, and the current at $114 \mu \mathrm{A}$. The field of volume was obtained at $25 \mathrm{~mm}$ with voxel 


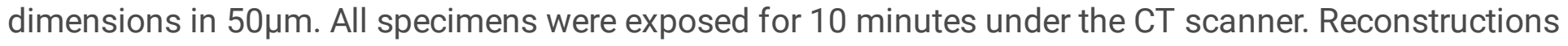
of the micro-CT image slices were performed with Mimics Research 21.0 software.

\section{Histomorphometric analysis of trabecular bone}

A total of 50 layers of scanning images were selected as the Region of Interest (ROI) to measure the microstructural trabecular parameters including bone mineral density(BMD, mg/cc), bone volume fraction (BV/TV , \%), connectivity density (Conn.D, $\mathrm{mm}^{\wedge-3}$ ), trabecular thickness (Tb.Th, mm) and trabecular separation (Tb.Sp, mm).

Subchondral trabecular region started below the subchondral plate and extended distally towards the metaphyseal trabecular, excluding both the cortical bone and growth plate interface. Subchondral bone parameters were analyzed by Analyze 12.0 software.

\section{Quantitative real-time polymerase chain reaction analysis}

For RNA isolation, approximately $20 \mathrm{mg}$ upper head part of the distal femur containing cartilago articularis was disrupted in liquid nitrogen utilizing mortar and pestle, and total RNA was isolated by Trizol (Beyotime, Shanghai, China) according to the manufacturer's protocol. The RNA yield was determined by measuring the absorbance at $260 \mathrm{~nm}$ and purity was assessed according to the ratio of absorbance readings at 260-280 nm using the NanoPhotometer NP80 (Implen GmbH, München, Germany). The RT First Strand cDNA Synthesis Kit (Servicebio, Wuhan, China) was used to generate complementary DNA using $1 \mu \mathrm{g}$ of total RNA. Quantitative real-time PCR was performed in a BIO-RAD CFX96 Real-Time PCR System (BIO RAD, California, USA) using the SYBR Green qPCR Master Mix (Servicebio, Wuhan, China). The fold changes in relative gene expression were calculated using the 2 $-\triangle \triangle \mathrm{Ct}$ method, with GAPDH serving as the housekeeping gene. Sequence-specific primers for complementary DNA (cDNA) amplified were shown in Table 1.

Table 1 Primer sequences of various rat genes used for qPCR

\begin{tabular}{|c|c|c|}
\hline Oligo name & Sequence $\left[5^{\prime}-3^{\prime}\right)$ & Fragment length $\square \mathrm{bp} \square$ \\
\hline TNF- $\alpha-F \square$ forward primer $\square$ & CCAGGTTCTCTTCAAGGGACAA & 80 \\
\hline TNF- $\alpha-R \square$ reverse primer $\square$ & GGTATGAAATGGCAAATCGGCT & \\
\hline GAPDH-F $\square$ forward primer $\square$ & CTGGAGAAACCTGCCAAGTATG & 138 \\
\hline GAPDH-R $\square$ reverse primer $\square$ & GGTGGAAGAATGGGAGTTGCT & \\
\hline MMP-13-F $\square$ forward primer $\square$ & ATGTGACACCTCTGAATTTTACCAG & 228 \\
\hline MMP-13-R $\rrbracket$ reverse primer $\square$ & CATGGGCAGCAACAATAAATAAG & \\
\hline Collagenase II-F $\square$ forward primer [ & GAGCGGAGACTACTGGATTGATC & 238 \\
\hline Collagenase II-R $\rrbracket$ reverse primer $\square$ & GACGTTAGCGGTGTTGGGAG & \\
\hline
\end{tabular}

\section{Western blot analysis}


Samples were homogenized and subsequently total proteins were extracted from distal femoral subchondral bone using RIPA lysis buffer (Beyotime, Shanghai, China) with 1\% PMSF. Total protein concentration in each sample was measured using a BCA Protein Assay Kit (Beyotime, Shanghai, China). The proteins $(20 \mu \mathrm{g} / \mathrm{lane})$ were separated on SDS-PAGE (8-10\% polyacrylamide) in tris-glycineSDS buffer $(2 \mathrm{~h}, 100 \mathrm{~V})$ and transferred to a PVDF membrane for $1.5 \mathrm{~h}$ at $300 \mathrm{~mA}$. Next, to avoid unspecific protein binding, the PVDF membranes were blocked for 10 minutes by QuickBlock ${ }^{\text {TM }}$ Blocking Buffer (Beyotime, Shanghai, China)at room temperature and incubated with primary antibodies in QuickBlock $^{\text {TM }}$ Primary Antibody Dilution Buffer (Beyotime, Shanghai, China) against-MMP13 (1:1000, Beyotime, AF7479), TNF-a (1:1000, Beyotime, AF8208), and $\beta$-actin (1:2000, Abmart, P30002) at $4{ }^{\circ} \mathrm{C}$ overnight. After washing for three times in tris buffered saline tween, the membranes were incubated for 1 $\mathrm{h}$ at room temperature with a horseradish peroxidase-conjugated secondary antibody (Beyotime, Shanghai, China). Finally, bands were visualized using enhanced chemiluminescence system G:BOX Chemi XRQ (Syngene, Cambridge, UK) the next day. Signal from positive bands was calculated relatively to the signal from the internal control ( $\beta$-actin-positive bands) using an imaging analysis system.

\section{IL-1 $\beta$ immunofluorescence}

Three sections from each knee were selected for IL-1 $\beta$ immunofluorescence. The deparaffinized and rehydrated sections were incubated with EDTA (PH8.0) antigen retrieval buffer in a microwave oven and kept warm for 15 minutes. After blocking with $3 \%$ bovine serum albumin, the sections were incubated with rabbit anti-rat IL-1 $\beta\left(1: 100\right.$, Beyotime, AF7209) overnight at $4{ }^{\circ} \mathrm{C}$. Next, the sections were incubated with Cy3 conjugated Goat anti-rabbit IgG (1:200, Servicebio, GB21303) at room temperature for 50 minutes. Finally, after the DAPI counterstain in nucleus, the sections were incubated with spontaneous fluorescence quenching reagent for about 5 minutes. All images were acquired at $400 \times$ magnification using a fluorescence microscope (Nikon E100, Tokyo, Japan) with Nikon DS-U3 software (Nikon, Tokyo, Japan).

\section{Statistical analyses}

Quantitative analysis of the Western blot bands was performed with Image J software (version 1.52, Wayne Rasband, USA). The data distribution was evaluated for normality with the Shapiro-Wilk test. The results are presented as the means \pm standard deviation (SD). Kruskal-Wallis nonparametric analyses were used to compare cartilage degeneration scores followed by the Dunn post hoc test. One-way analysis of variance followed by the LSD post hoc test was used to determine statistical differences. The data were analyzed using GraphPad Prism 8.0 software (San Diego, USA). P values less than 0.05 were considered statistically significant.

\section{Result}

\section{Histopathology in MIA-induced OA rats}


To understand the changes of articular cartilage after MIA modeling, the articulation of the animals that received MIA was investigated under histological observations at different stages of modeling. The cartilage histology was evaluated in all groups on day 28 and representative micrographs were shown in Fig. 1.

Saline-injected group did not show any remarkable lesions in the articular cartilage. The histological sections showed that the proximal tibias of the rats in the sham group were well-stained and the cartilage layer was intact. The knee joint of the rats receiving MIA was denuded and erosive compared to the sham group. MIA-injected animals showed erosion damage in the articular cartilage of the bilateral tibial plateau. Injuries were more severe in the four weeks group, predominantly in the junction of the femoral condyles, due to the greater contact between the tibiofemoral structures. Simultaneously, a rough surface with fibrous degeneration was presented in partial thinness lesion district of cartilage, with an extensive area covered with fibrous tissue. Moreover, accompanied by the absence of safranin $\mathrm{O}$ and toluidine blue staining, MIA injected animals underwent maximum loss in the knee joint region compared to salinetreated sham animals with notable aggrecan loss and disappearance of chondrocytes at the deep zone of articular cartilage.

As shown in Fig. 2, the OOCHAS scores of the MIA-injected group were significantly higher than those of the sham group, which was $0(0,1)$. Simultaneously, treatment with MIA exacerbated the degenerative changes significantly as the duration of injection extended $(p<0.05)$. By week two, the MIA induced joints exhibited moderate cartilage degeneration and the OOCHAS score was 12(9.75,15). By week four, severe cartilage degeneration occurred and the OOCHAS score was $24(20,24)$.

\section{Joint injury caused by MIA intra-articular injection}

As shown in Fig. 3A, Micro-CT three-dimensional reconstructed images confirmed that the articular cartilage in the sham group presented with normal morphology and a smooth surface, while the MIAinjected groups exhibited a rough surface in the cartilage, with an irregular morphological structure and osteophyte formation. Detailed analysis of tibial bone by Micro-CT indicated that MIA degraded cartilage substantially relative to the sham group. Moreover, the MIA-injected groups had an extensive chondrocyte degeneration in the tibial plateau, collapse of necrotic articular cartilage, and an extensive area of cartilage defects with the extension of time. Furthermore, after MIA injection, Micro-CT analysis revealed a significant loss in subchondral bone parts in MIA-injected groups (Fig. 3B and Fig. 3C). The subchondral bone in the tibial plateau showed increased collapse and cracks of bone trabeculae and loss of reticular structure compared to the intact and regular trabecular network of the sham group. In addition, thinning of the subchondral bone trabeculae was observed by Micro-CT two-dimensional and three-dimensional sectional view, together with widening of trabecular separation. Injection of MIA at the dose of $3 \mathrm{mg}$ showed significantly changes in the trabecular structure of subchondral bone, articular surface cartilage, as well as in bone mineral density, which gradually aggravated degradation with the progression of modeling time.

\section{Histomorphometric analysis of trabecular bone}


Intra-articular injection of MIA not only leaded to damage of articular cartilage but also increased bone remodelling followed by subchondral bone deterioration with the progression of the disease. Bone mineral density and trabecular thickness were significantly declined in the subchondral bone of rat tibia treated with MIA at both week two and week four, simultaneously aggravated osteoporosis was observed over time, compared to the sham group (Fig. 4). Results of Micro-CT at the subchondral bone site showed that MIA induction decreased BV/TV to $(30.72 \pm 5.122) \%$ in MIA two-week group and $(25.81 \pm 2.608) \%$ in MIA four-week group compared to $(38.22 \pm 3.284) \%$ in sham group. Decrease in subchondral BV/TV after MIA induced OA ultimately led to the reduction of BMD which was $(1330.66 \pm 38.99) \mathrm{mg} / \mathrm{cc}$ in MIA two weeks group and $(1285.5 \pm 47.08) \mathrm{mg} / \mathrm{cc}$ in MIA four weeks group compared to $(1367.97 \pm 37.43) \mathrm{mg} / \mathrm{cc}$ in sham group (Fig. 4). Tb. Th in sham group was $(0.1912 \pm 0.0293) \mathrm{mm}$, decreased to $(0.1750 \pm 0.0194) \mathrm{mm}$ in MIA two weeks group, and decreased to $(0.1414 \pm 0.0299) \mathrm{mm}$ in MIA four weeks group (Fig. 4). MIA significantly thinned bone trabeculae in bone as assessed by Tb.Th that leads to higher trabecular separation in the MIA rats, which was indicated by a significantly increase to $(0.4750 \pm 0.0903) \mathrm{mm}$ in MIA two weeks group and $(0.5307 \pm 0.0893) \mathrm{mm}$ in MIA four weeks group compared to $(0.4288 \pm 0.0710) \mathrm{mm}$ in sham group(Fig. 4). On the other hand, trabecular connectivity decreased from $(57.94 \pm 8.050) \mathrm{mm}^{-3}$ in the sham group to $(52.39 \pm 8.781) \mathrm{mm}^{-3}$ in MIA two weeks group and to $(45.48 \pm 10.14) \mathrm{mm}^{-3}$ in MIA four weeks group (Fig. 4).

\section{Effects of MIA on the expression of inflammatory cytokines and cartilage associated proteins in the articular cartilage region of knee joints}

Quantitative real-time PCR and Western blot semiquantitative analysis were used to detect the expression of proinflammatory and cartilage associated proteins. The progressive destruction of articular cartilage was caused by MIA injection that significantly upregulated the expression of proinflammatory genes IL$1 \beta$, TNF-a, matrix metalloproteinase 13(MMP-13) and downregulate the expression of cartilage specific protein collagen II (Fig. 5). When we administered MIA for a long time, we observed aggravation of the deleterious effects of MIA. MIA increased the expression of IL-1 $\beta$ by approximately 7 folds in MIA twoweek group, 16 folds in MIA four-week group (Fig. 5A), and moreover, upregulated the expression of TNFa by approximately 6 folds in MIA two-week group, 16 folds in MIA four-week group (Fig. 5B) compared to sham group. These cytokines further damaged the joints by enhancing the expression of MMP-13 (Fig. 5C). In addition to these harmful effects, MIA blocked chondrogenic activity by down-regulating the synthesis of extracellular matrix (ECM) components like collagen II by approximately $70 \%$ in MIA two week group, 87\% in MIA four weeks group (Fig. 5D) compared to the sham group. These differences in expression were also verified by Western blot semiquantitative analysis (Fig. 6). The expression of TNF-a and MMP-13 increased gradually with the increase of modeling time, which was significantly different compared with the sham group (Fig. 6).

\section{Immunofluorescence}

As indicated by the immunofluorescence images (Fig. 7), IL-1 $\beta$ of the tibial plateau was sparsely expressed in the sham group. However, compared with the saline injection joints, the expression of IL-1 $\beta$ 
was significantly improved by the MIA treatment. With the prolonging of modeling time, the expression level increased gradually.

\section{Discussion}

$\mathrm{OA}$ is a degenerative joint disease that leads to functional disability and progressive loss of articular cartilage induced by diverse factors. In this study, we examined the impact of OA on the cartilage and subchondral bone of the knee joints in rodent models. OA in rats was induced by a widely used toxic modeling reagent, MIA, which causes apoptosis of chondrocytes, subchondral bone changes, cartilage erosion, osteophytes and cartilage clefts ${ }^{[18]}$.

$\mathrm{OA}$ affects the subchondral bone due to the aberrant mechanical loading, which will cause microfractures in the subchondral bone and the associated increased bone resorption remodeling activity ${ }^{[19]}$. Increased osteoclastic activity and bone resorption are often observed in the subchondral bone during the early stages of OA. At later stages, the bone remodeling activity tends to bone formation, leading to abnormal bone formation and osteophytes ${ }^{[20,21]}$. It has been confirmed that microstructural changes in the subchondral bone environment are relevant to OA-related cartilage degeneration through cartilage-bone cross-talk ${ }^{[22]}$. Subchondral bone exerts important shock-absorbing functions for the overlying articular cartilage, attenuating approximately $30 \%$ of the joint load during movement ${ }^{[23]}$ and experiences a constant adaptation in response to changes in the mechanical environment through modelling or remodelling ${ }^{[24]}$. Therefore, the overall properties of the subchondral bone were evaluated in this study.

Traumatic OA modeling by anterior cruciate ligament excision was found a decrease in trabecular thickness and bone trabecular number at the early stage, along with an increase in bone resorption resulting in a diminution in bone mineral density. In the late stage, although there was increased bone formation in the subchondral bone and osteophyte, loss of cancellous bone and thinning of bone trabeculae was observed ${ }^{[25]}$. And again, in the inflammatory OA model, the subchondral bone plate was found to be thinner as time goes by, with increased porosity, trabecular loss, and reduced bone mass ${ }^{\text {[26] }}$. In parallel with these findings, our results demonstrate that the trabecular separation was increased in the MIA-treated rats compared to the sham group. The corresponding decrease in bone mineral density, connectivity density, trabecular thickness, and bone volume fraction was also observed with statistical significance. These observations suggest the increase in bone resorption activity due to OA.

Previous studies support that both the cartilage and subchondral bone communicate to each other through biomechanical pathways for maintaining the homeostasis of the joint environment. In this research, histopathological examination showed that MIA induced remarkable lesions in OA, including chondrocyte degeneration, collapse and fragmentation of subchondral bone, loss of chondrocytes and a focally extensive area of cartilage loss, which was confirmed by safranin-o and toluidine blue staining. MIA produced extensive damage to the joint cartilage, corroborating previous findings ${ }^{[1,14]}$. Immediately after the injection of MIA, a robust inflammatory response was identified in the joint through histology ${ }^{[27]}$. 
Moreover, defects in articular cartilage increase various inflammatory cytokines and decrease chondrocytes synthesis that further leads to increased bone remodelling and subchondral bone deterioration. MIA injection was able to trigger degenerative changes of the joint, pathological morphologic changes in articular cartilage as indicated by the higher scores for all histological aspects evaluated. Thereupon, the subsequent release of cartilage destruction products can give rise to localized inflammation of the joint. As a result, the self-perpetuating inflammation will cause more cartilage to breakdown ${ }^{[5,6]}$.

On the basis of these results, we sought to determine whether transformations in subchondral bone structure are associated with cartilage inflammatory response in rat chondrocytes. However, there is no consensus on the relationship between subchondral bone structure modifications and inflammatory responses in MIA induced OA.

Previous studies have reported that intra-articular injection of MIA initially causes severe and acute inflammation, which gives rise to degenerative changes in the cartilage ${ }^{[15]}$. It has been recognized that inflammation is a dominant biological event related to the pathogenesis of $\mathrm{OA}^{[28]}$. Although the precise mechanism of $\mathrm{OA}$ remains uncertain, a growing body of literature suggests that inflammatory factors exert a crucial action in the downstream inflammatory cascade ${ }^{[29]}$. Previous research reported that inflammatory cytokines and other markers are found higher in OA patients ${ }^{[30]}$. Secreted inflammatory cytokines, such as IL-1 $\beta$ and TNF- $\alpha$, are primary instigators in the regional and systemic inflammatory processes of $O A^{[31]}$. As a critical inflammatory cytokine, IL-1 $\beta$ is associated with the pathological progression of $O A$.

To determine whether the structural breakdown of cartilage and the inflammatory reaction caused by MIA aggravated with time, the level of IL-1 $\beta$ and TNF-a was quantified and compared. In this study, using our established MIA-induced rat model, the expression quantity of IL-1 $\beta$ and TNF-a was also found to be higher in MIA-treated rats compared to the sham group, comparable to the observation in previous MIA OA models.

Pro-inflammatory cytokines, such as IL-1 $\beta$, are known to mediate the degradation of matrix proteins, such as aggrecan and collagen, by upregulate the gene expression level of MMPs ${ }^{[32]}$ and apoptosis in chondrocytes $^{[33]}$. Additionally, many studies have confirmed that IL-1 $\beta$ is co-expressed with OA-related MMPs, resulting in cartilage degradation ${ }^{[34]}$. MMPs are mainly in charge of the degradation of arthritic cartilage and remodeling of ECM during growth, of which type II collagen is the key component ${ }^{[35]}$.

MMP-13 is known as collagenase-3 and is increased during the process of early-onset OA. MMP-13 can inhibit the synthesis of collagen and degrade components of the ECM ${ }^{[3,36]}$. It is also known that MMP13 is highly expressed in the articular cartilage of human OA patients.

Molecular analysis by qRT-PCR and Western blot revealed that the imbalance between the cartilage anabolic and inflammation-related genes might account for the advanced condylar destruction and 
chondrocyte apoptosis following MIA induction. As shown by the data, MIA affected the joints by enhancing the expression of MMP-13, which had a destructive effect on cartilage. These metalloproteinases block chondrocyte activity by down-regulating the synthesis of ECM components,

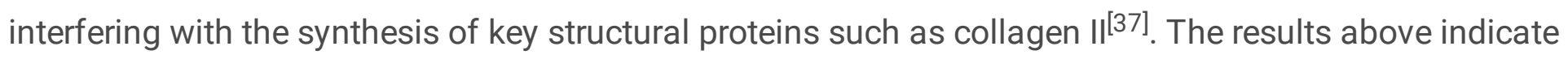
that MIA exerts a strong proinflammatory effect on chondrocytes.

Taken collectively, morphological observations demonstrated that MIA substantially deteriorated articular cartilage as evidenced by irregular surfaces, thinning, and necrotic areas and increased OOCHAS score in MIA-injected OA rats, indicating aggravation of cartilage damage. Additionally, the application of MIA accelerated the expression of representative inflammatory cytokines like IL-1 $\beta$, TNF-a, in osteoarticular chondrocytes in our OA model, further indicating a strong inflammatory effect of MIA. Furthermore, given the structural parameters of subchondral trabecular bone seen in vitro, we hold the opinion that that intraarticular injection of MIA can simulate subchondral bone structure changes during the acute phase of OA.

\section{Conclusions}

These findings indicated that MIA treatment increases the MIA induced inflammation, articular and subchondral bone loss. However, a limitation that confirms the effect of MIA on OA modeling as observed by us is the progressive degenerative nature of the disease in humans where extensive chondrocyte loss and cartilage degeneration in inflammatory $O A$ are impossible to reverse or modify. In the early stages of OA that follow a traumatic injury, chondrocytes enter a hypertrophic state as a type of compensatory mechanism to modify the extracellular matrix in response to increased load ${ }^{[38]}$. This pathological signaling starts at the site of injury and spreads throughout the articular surface leading to an almost full chondrocyte loss and complete degeneration of the articular surface ${ }^{[38]}$. As a result, inducing the formation of OA with MIA treatment may not have the same end-stage manifestations such as subchondral osteosclerosis that we observe when OA occurs naturally, slowly and progressively.

\section{Abbreviations}

OA: Osteoarthritis; IL-1ß: Interleukin-1 beta; TNF-a: Tumor necrosis factor alpha; MMPs: Matrix metalloproteinases; MIA: Monosodium iodoacetate; GAPDH:Glyceraldehyde-3-phosphate dehydrogenase; H\&E: Hematoxylin and eosin; Micro-CT: Micro computed tomography; ROI: Region of Interest; BMD: Bone mineral density; BV/TV: Bone volume fraction; Conn.D: Connectivity density; Tb.Th: Trabecular thickness; Tb.Sp: Trabecular separation; cDNA: complementary DNA; EDTA: Ethylene diamine tetraacetic acid; ECM: extracellular matrix.

\section{Declarations}

\section{Ethics approval and consent to participate}


The experiments were performed in line with the recommendations of the Ethics Committee of Chinese PLA General Hospital, which approved the experimental protocol (Approval numberSQ2020127).

\section{Availability of data and materials}

The datasets used and analyzed during the current study are available from the corresponding author on reasonable request.

\section{Competing interests}

The authors report no competing interests.

\section{Funding}

This work was carried out with the support of "Health Bureau of Logistical Support Department of CMC (Project No.18BJZ23)". Funding body has been used in the collection, analysis, and interpretation of data and in writing the manuscript.

\section{Author contributions}

Zheming Bao, Mengli Chen: conception and design, acquisition of data, analysis and interpretation of data, drafting of the manuscript, critical revision of the manuscript and final approval of the manuscript; Chen Li, Qing Shan: acquisition of data and final approval of the manuscript; Yichen Wang, Wenshan Yang: analysis and interpretation of data, final approval of the manuscript.

\section{Acknowledgements}

Not applicable.

\section{Author details}

Zheming Bao1,2, E-mail: baozhemingdyx@163.com, postal address: 105 Jingliu Road, Jinan City, Shandong Province, China; Mengli Chen ${ }^{1}$, Correspondence author, E-mail: hellolily301@163.com; Chen Li ${ }^{1}$, E-mail: 18301116236@163.com; Qing Shan ${ }^{1}$, E-mail: 264798706@qq.com; Yichen Wang ${ }^{1}$, E-mail: wangyichen0701@126.com; Wenshan Yang ${ }^{1}$, E-mail: 459438265@qq.com.

1. Pharmacy Department, Chinese PLA General Hospital, Beijing, China 2. Orthopedics department, 960th Hospital of PLA Joint Service Support Force, Jinan, China.

\section{References}

1. Moon SJ, Park SH, Kim HY, et al. Rebamipide attenuates pain severity and cartilage degeneration in a rat model of osteoarthritis by downregulating oxidative damage and catabolic activity in chondrocytes[J]. Osteoarthritis \& Cartilage, 2012, 20(11): pp. 1426-38. 
2. Lampropoulou-Adamidou K, Karadimas EV, Liakou C, et al. Useful animal models for the research of osteoarthritis[J]. European Journal of Orthopaedic Surgery \& Traumatology, 2014, 24(3).

3. Liu-Bryan R. Inflammation and intracellular metabolism: new targets in OA[J]. Osteoarthritis Cartilage. 2015;23(11):1835-42.

4. Kelwick R, Desanlis I, Wheeler GN, et al. The ADAMTS (A Disintegrin and Metalloproteinase with Thrombospondin motifs) family[J]. Genome Biol. 2015;16(1):113.

5. Mary G. Miguel, et al. Inflammation in osteoarthritis[J]. Current Opinion in Rheumatology; 2011.

6. Sokolove J, Lepus CM. Role of inflammation in the pathogenesis of osteoarthritis: latest findings and interpretations[J]. Therapeutic Advances in Musculoskeletal Disease. 2013;5(2):77.

7. Tat SK, Lajeunesse D, Pelletier JP, et al. Targeting subchondral bone for treating osteoarthritis: what is the evidence?[J]. Best Pract Res Clin Rheumatol. 2010;24(1):51-70.

8. Casta?Eda S, Roman-Blas JA, Largo R, et al. Subchondral bone as a key target for osteoarthritis treatment[J]. Biochem Pharmacol. 2012;83(3):315-23.

9. Sanchez C, Deberg MA, Piccardi N, et al. Subchondral bone osteoblasts induce phenotypic changes in human osteoarthritic chondrocytes[J]. Osteoarthritis Cartilage. 2005;13(11):988-97.

10. Prasadam I, Gennip SV, Friis T, et al. ERK-1/2 and p38 in the regulation of hypertrophic changes of normal articular cartilage chondrocytes induced by osteoarthritic subchondral osteoblasts[J]. Arthr Rhuem. 2014;62(5):1349-60.

11. Goldring MB, Goldring SR. Articular cartilage and subchondral bone in the pathogenesis of osteoarthritis[J]. 1192: Annals of the New York Academy of Sciences; 2010. pp. 230-7.

12. Kim WK, Chung HJ, Pyee $Y$, et al. Effects of intra-articular SHINBARO treatment on monosodium iodoacetate-induced osteoarthritis in rats[J]. Chinese Medicine, 2016, 11(1).

13. Tomoya U, Andrea, et al. The Chondroprotective Role of Erythromycin in a Murine Joint Destruction Model[J]. Cartilage, 2016.

14. Nwosu LN, Mapp PI, Chapman V, et al. Relationship between structural pathology and pain behaviour in a model of osteoarthritis (OA)[J]. Osteoarthritis Cartilage. 2016;24(11):1910-7.

15. Yamada EF, Salgueiro AF, Goulart ADS, et al. Evaluation of monosodium iodoacetate dosage to induce knee osteoarthritis: Relation with oxidative stress and pain[J]. International Journal of Rheumatic Diseases; 2018.

16. Schmitz N, Laverty S, Kraus VB, et al. Basic methods in histopathology of joint tissues[J]. Osteoarthritis Cartilage. 2010;18(Suppl 3):113-6.

17. Pritzker KP, Gay S, Jimenez SA, et al. Osteoarthritis cartilage histopathology: grading and staging[J]. Osteoarthritis Cartilage. 2006;14(1):13-29.

18. Guzman RE, Evans MG, Bove S, et al. Mono-iodoacetate-induced histologic changes in subchondral bone and articular cartilage of rat femorotibial joints: an animal model of osteoarthritis[J]. Toxicol Pathol. 2003;31(6):619-24. 
19. Kothari A, Guermazi A, Chmiel JS, et al. Within-subregion relationship between bone marrow lesions and subsequent cartilage loss in knee osteoarthritis[J]. Arthritis Care Res (Hoboken). 2010;62(2):198-203.

20. Li G, Yin J, Gao J, et al. Subchondral bone in osteoarthritis: insight into risk factors and microstructural changes[J]. Arthritis Research \& Therapy, 2013, 15(6).

21. Yuan XL, Meng HY, Wang YC, et al. Bone-cartilage interface crosstalk in osteoarthritis: potential pathways and future therapeutic strategies[J]. Osteoarthritis Cartilage. 2014;22(8):1077-89.

22. Goldring SR, Goldring MB. Changes in the osteochondral unit during osteoarthritis: structure, function and cartilage-bone crosstalk[J]. Nature Reviews Rheumatology, 2016.

23. Imhof H, Sulzbacher I, Grampp S, et al. Subchondral bone and cartilage disease: a rediscovered functional unit[J]. Invest Radiol. 2000;35(10):581-8.

24. Zhen G, Wen C, Jia X, et al. Inhibition of TGF- $\beta$ signaling in mesenchymal stem cells of subchondral bone attenuates osteoarthritis[J]. Nat Med. 2016;19(6):704-12.

25. Intema F, Hazewinkel HaW, Gouwens D, et al. In early OA, thinning of the subchondral plate is directly related to cartilage damage: results from a canine ACLT-meniscectomy model[J]. Osteoarthritis Cartilage. 2010;18(5):691-8.

26. Siebelt M, Groen HC, Koelewijn SJ, et al. Increased physical activity severely induces osteoarthritic changes in knee joints with papain induced sulfate-glycosaminoglycan depleted cartilage[J]. Arthritis Res Ther. 2014;16(1):R32.

27. Udo M, Muneta T, Tsuji K, et al. Monoiodoacetic acid induces cartilage degeneration and synovitis in rats in a dose- and time- dependent manner[J]. Osteoarthritis Cartilage. 2016;24:405-6.

28. Vina ER, Kwoh CK. Epidemiology of osteoarthritis: literature update[J]. Current Opinion in Rheumatology; 2018.

29. Kapoor M, Martel-Pelletier, et al. Role of proinflammatory cytokines in the pathophysiology of osteoarthritis[J]. Nature Reviews Rheumatology, 2011.

30. Ziskoven C, Jäger M, Zilkens C, et al. Oxidative stress in secondary osteoarthritis: from cartilage destruction to clinical presentation?[J]. Orthopedic Reviews. 2010;2(2):e23.

31. Chevalier X, Conrozier T, Richette P. Desperately looking for the right target in osteoarthritis: the antiIL-1 strategy[J]. Arthritis research \& therapy, 2011, 13(4): p. 124.

32. Goldring MB, Otero M, Plumb DA, et al. Roles of inflammatory and anabolic cytokines in cartilage metabolism: signals and multiple effectors converge upon MMP-13 regulation in osteoarthritis[J]. Eur Cell Mater. 2011;21:202-20.

33. Wang M, Sampson ER, Jin $\mathrm{H}$, et al. MMP13 is a critical target gene during the progression of osteoarthritis[J]. Arthritis research \& therapy, 2013, 15(1): p. R5.

34. Aida Y, Maeno M, Suzuki N, et al. The effect of IL-1 $\beta$ on the expression of matrix metalloproteinases and tissue inhibitors of matrix metalloproteinases in human chondrocytes[J]. Life Sci. $2005 ; 77(25): 3210-21$. 
35. Klein T, Bischoff R. Physiology and pathophysiology of matrix metalloproteases[J]. Amino Acids. 2011;41(2):271-90.

36. Chen JJ, Huang JF, Du WX, et al. Expression and significance of MMP3 in synovium of knee joint at different stage in osteoarthritis patients[J]. Asian Pacific Journal of Tropical Medicine. 2014;7(004):297-300.

37. Dai L, Xin Z, Hu X, et al. Silencing of microRNA-101 prevents IL-1 $\beta$-induced extracellular matrix degradation in chondrocytes[J]. Arthritis research \& therapy, 2012, 14(6): p. R268.

38. Kraan PD, Berg W. Chondrocyte hypertrophy and osteoarthritis: role in initiation and progression of cartilage degeneration?[J]. Osteoarthritis \& Cartilage, 2012, 20(3): pp. 223-32.

\section{Figures}
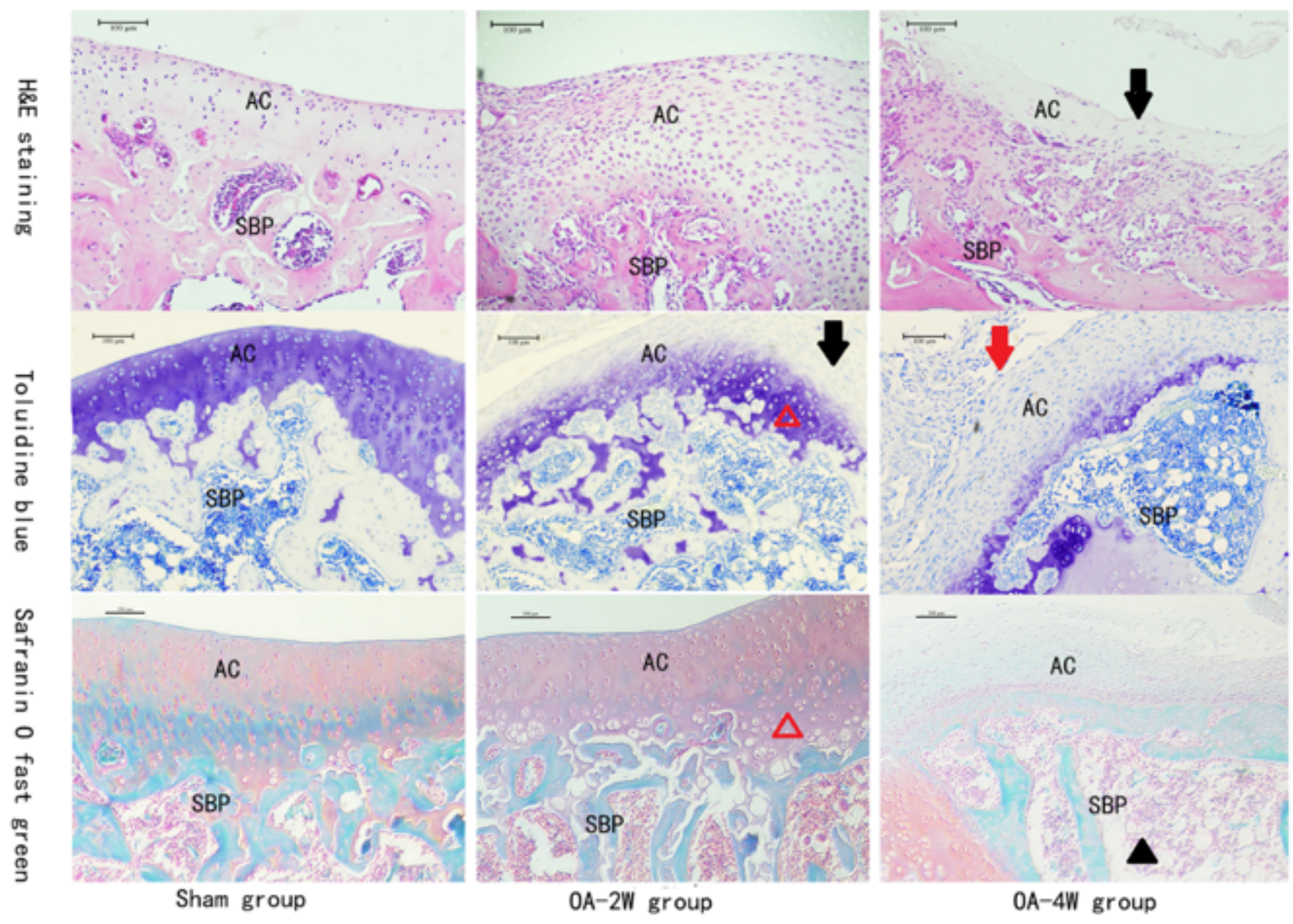

\section{Figure 1}

Representative micrographs of rat tibial joints receiving different treatments. Red arrow: a rough surface with fibrous degeneration; Black arrow: absence of staining in cartilage surface; Black triangle: increased trabecular space; Red triangle: hypertrophy and vacuolated chondrocytes Abbreviation: SBP: subchondral bone plate; AC, articular cartilage. Sham group: animals received sterile saline $0.9 \%$ for four weeks; OA- 
2W group: animals received MIA for two weeks; OA-4W group: animals received MIA for four weeks. Scale bar, $100 \mu \mathrm{m}$.

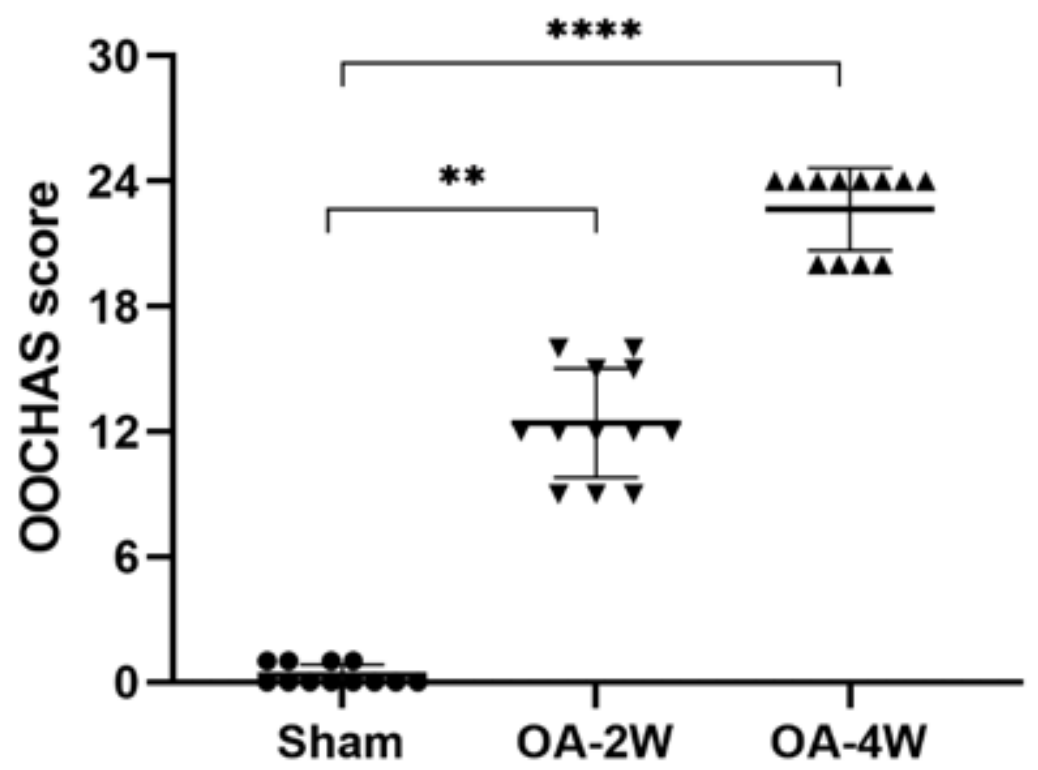

Figure 2

OOCHAS scores in the three groups. $n=12$ per group. ${ }^{\star *} \mathrm{P}<0.01$ compared to the sham group and $* \star \star \star$ $\mathrm{P}<0.0001$ compared to the sham group. Sham: animals received sterile saline $0.9 \%$ for four weeks; OA$2 \mathrm{~W}$ : animals received MIA for two weeks; OA-4W: animals received MIA for four weeks.
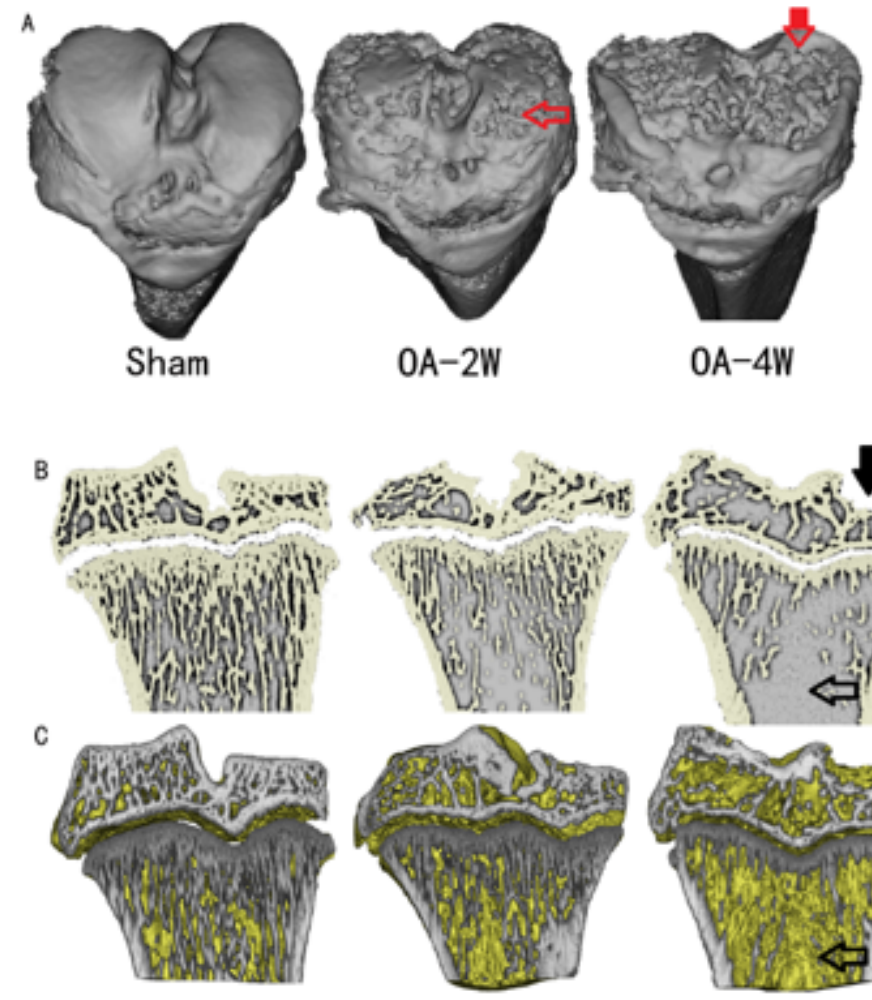

Sham

$0 \mathrm{~A}-2 \mathrm{~W}$

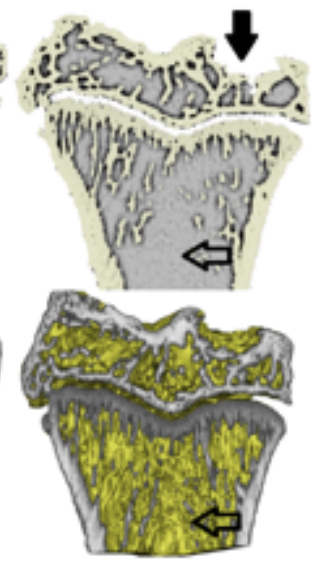

$0 A-4 W$ 


\section{Figure 3}

(A)Representative micro-CT three-dimensional images of rat tibial bone in three groups. (B)Representative micro-CT two-dimensional sectional view of knee. (C)Representative micro-CT three-dimensional sectional view of knee. Red arrow: The MIA groups exhibited a rough surface on the cartilage and an extensive area of cartilage defects. Black arrow: There was significant cartilage loss and trabeculae missing together with widening of trabecular separation in MIA-injected group. Sham: animals received sterile saline $0.9 \%$ for four weeks; OA-2W: animals received MIA for two weeks; OA-4W: animals received MIA for four weeks.
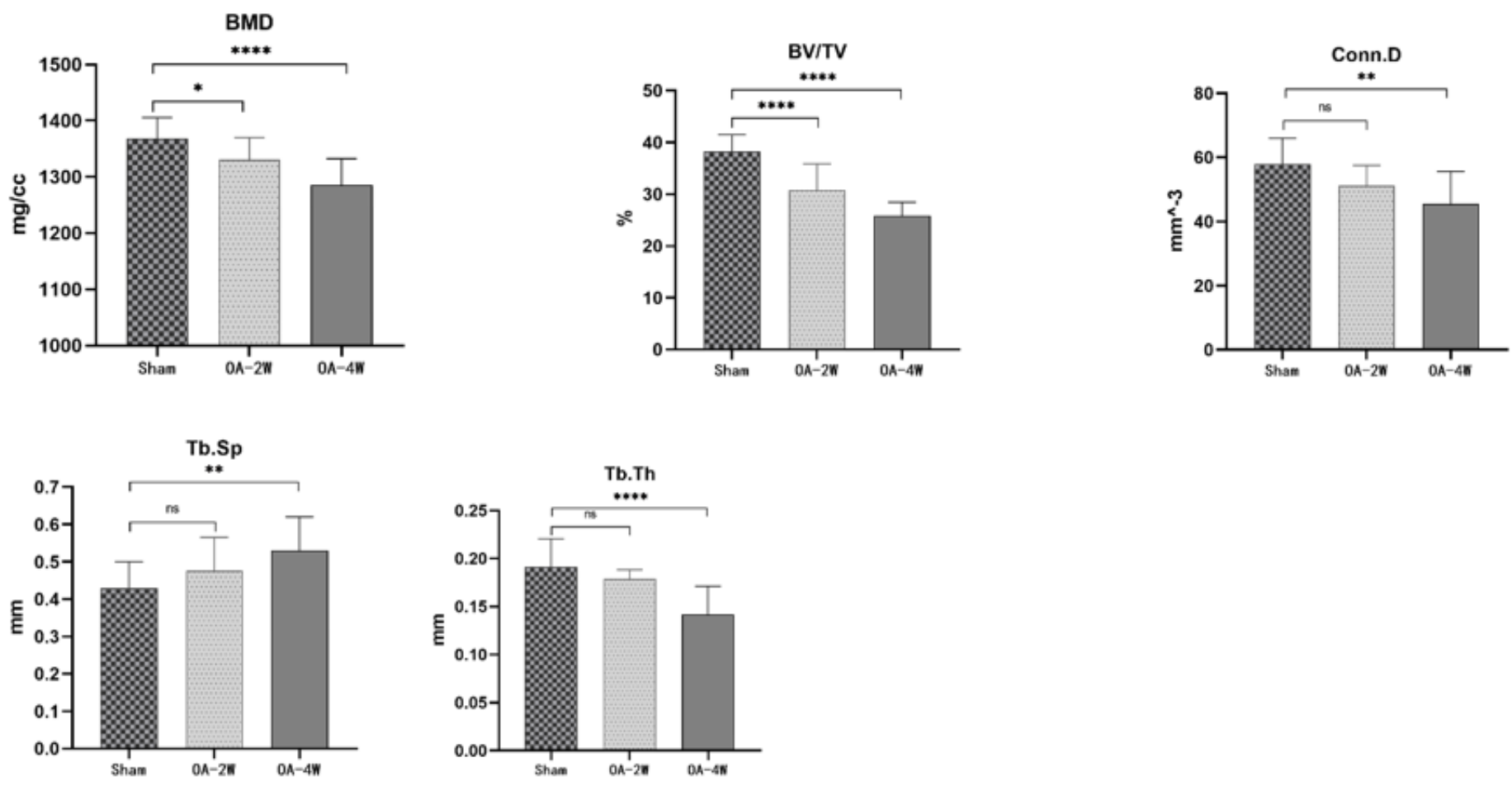

\section{Figure 4}

Quantitative micro-CT analysis of various parameters of tibial subchondral bone. Bone mineral density (BMD), bone volume/tissue volume (BV/TV), trabecular thickness ( $\mathrm{Tb}$. Th), trabecular separation ( $\mathrm{Tb}$. $\mathrm{SP}$ ), and connectivity density (Conn. D). $\mathrm{n}=9$ per group. ${ }^{*} \mathrm{P}<0.05$ compared to the sham group, ${ }^{*} \mathrm{P}<$ 0.01 compared to the sham group and $\star \star \star \star ~ P<0.0001$ compared to the sham group, ns stands for no statistical significance compared to the sham group. Sham: animals received sterile saline $0.9 \%$ for four weeks; OA-2W: animals received MIA for two weeks; OA-4W: animals received MIA for four weeks. 

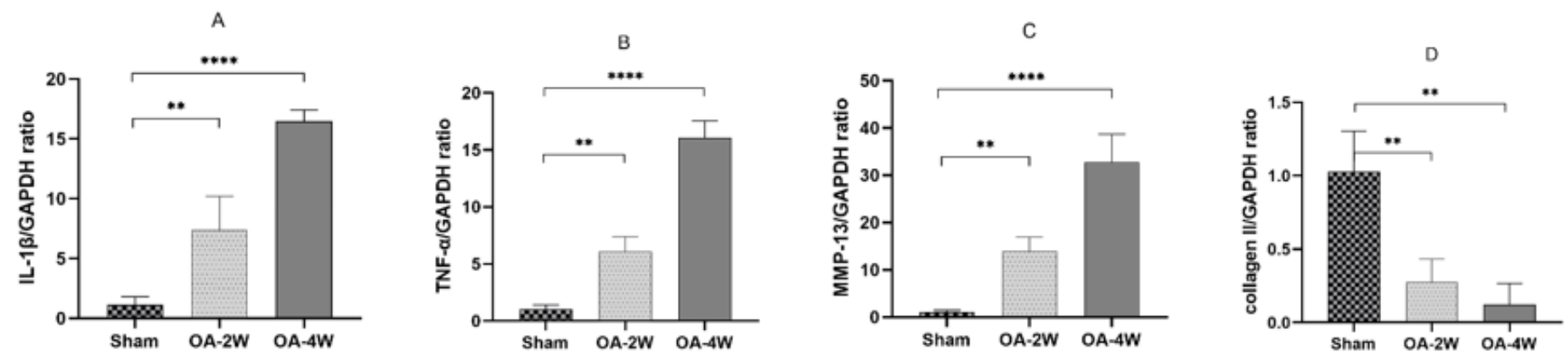

Figure 5

Relative mRNA expression of genes (A) IL1- $\beta$, (B) TNF- $\alpha$, (C) MMP-13, (D) Collagen囚in distal femur containing cartilage and subchondral region. All values are expressed as Mean $\pm S D$. ( $n=9 /$ group); **P<

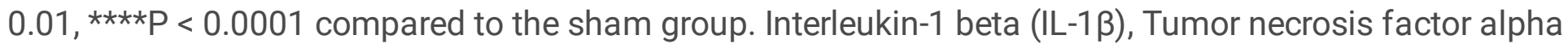
(TNF-a), Matrix metalloproteinase 13(MMP-13). Sham: animals received sterile saline $0.9 \%$ for four weeks; OA-2W: animals received MIA for two weeks; OA-4W: animals received MIA for four weeks.
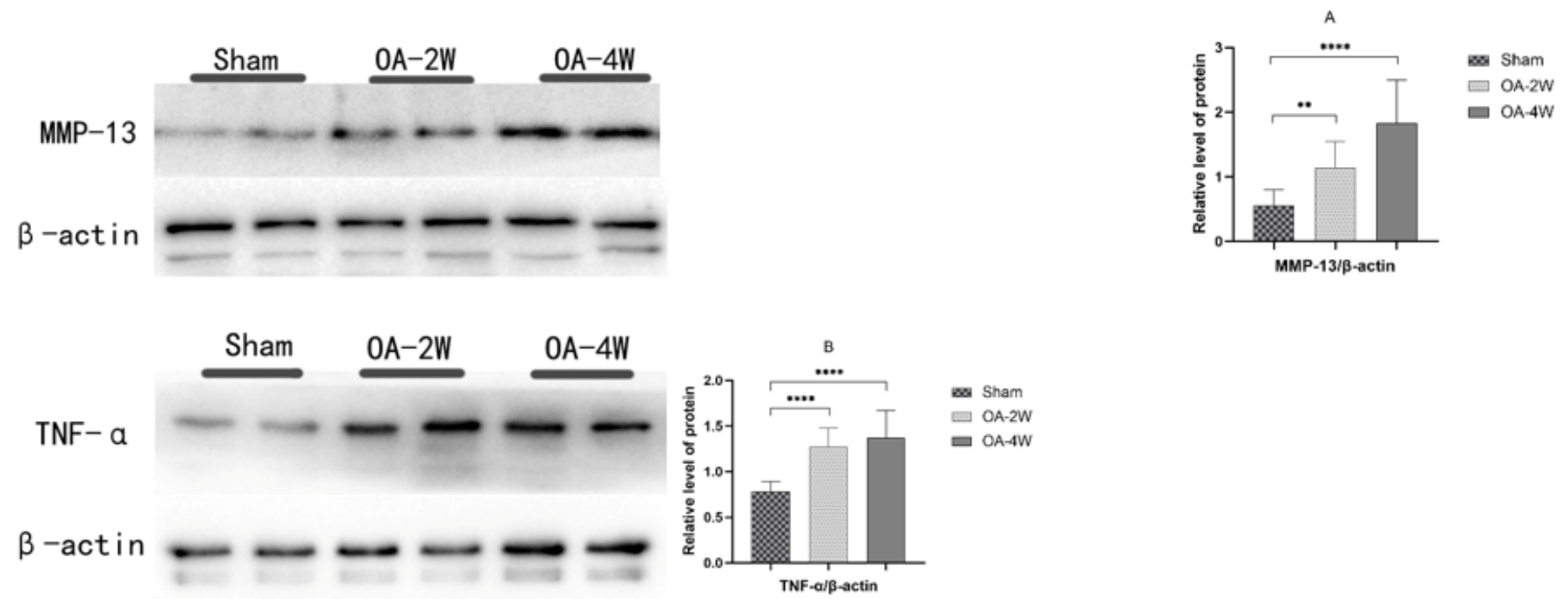

\section{Figure 6}

Effect of MIA treatment on cartilage inflammatory and degradative proteins. Western blot analysis of (A) MMP-13, (B) TNF-a in the distal femur containing cartilage and subchondral region is represented after treatment in rat articular. All values are expressed as Mean \pm SD. $(n=6 /$ group $) ;{ }^{\star *} P<0.01,{ }^{\star \star \star *} P<0.0001$ compared to the sham group. Tumor necrosis factor alpha (TNFa), Matrix metalloproteinase 13(MMP13). Sham: animals received sterile saline $0.9 \%$ for four weeks; OA- $2 \mathrm{~W}$ : animals received MIA for two weeks; OA-4W: animals received MIA for four weeks. 


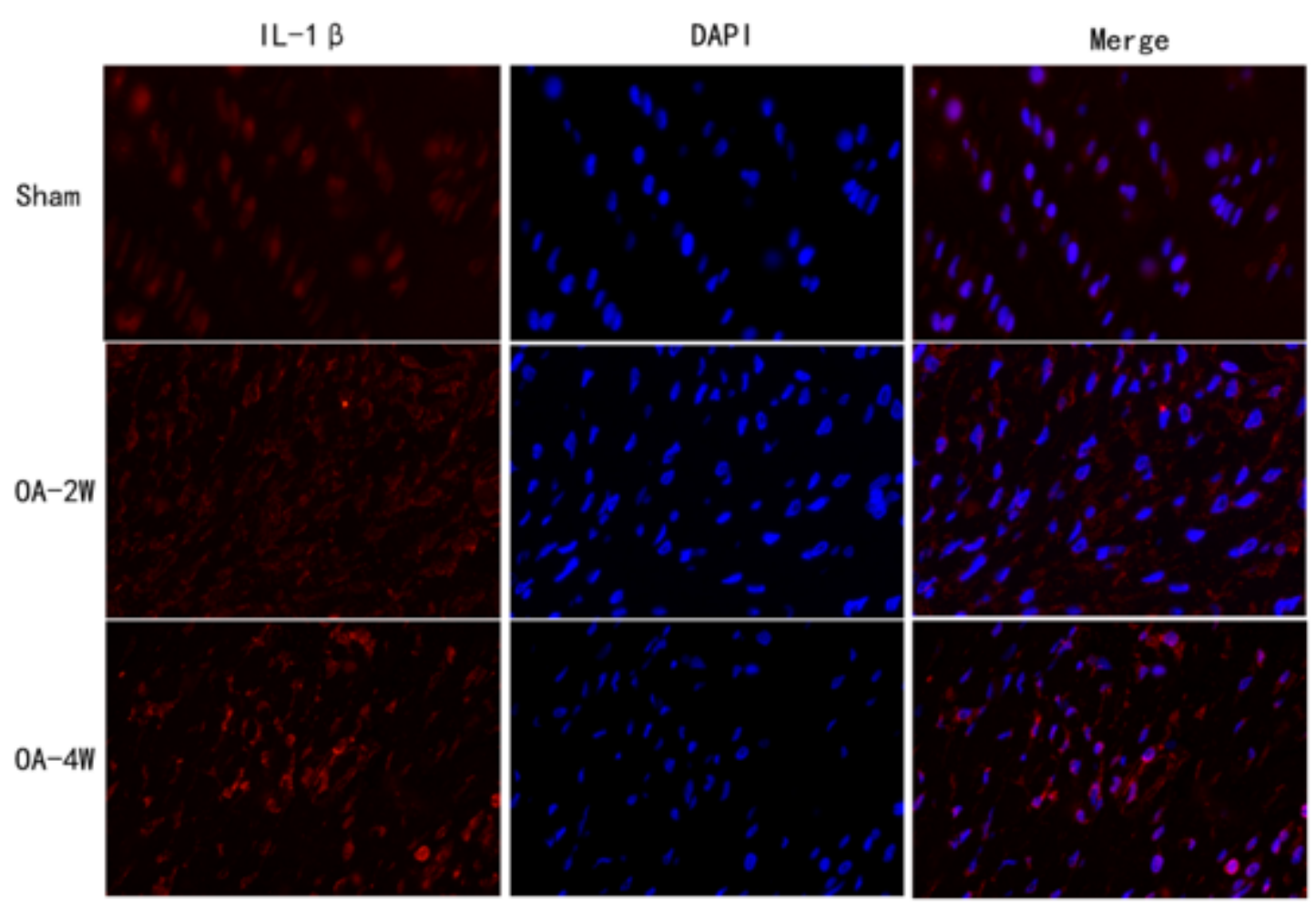

\section{Figure 7}

Effects of MIA treatment on the expression of IL-1 $\beta$ in the tibial plateau. Vision: $400 \times$ magnification. IL-1 $\beta$ of the tibial plateau was sparsely expressed in the sham group. The expression of IL-1 $\beta$ was improved by MIA treatment with the prolonging of modeling time. Sham: animals received sterile saline $0.9 \%$ for four weeks; OA-2W: animals received MIA for two weeks; OA-4W: animals received MIA for four weeks. 